\title{
Rheumatoid arthritis Fibroblast-like synoviocytes maintain tumor-like biological characteristics through ciRS-7-dependent regulation of miR-7
}

\section{Zuoyu Hu}

Third Affiliated Hospital of Sun Yat-Sen University

Jingrong Chen

Third Affiliated Hospital of Sun Yat-Sen University

Manli Wang

Third Affiliated Hospital of Sun Yat-Sen University

\section{Weizhen Weng}

Third Affiliated Hospital of Sun Yat-Sen University

\section{Ye Chen}

Third Affiliated Hospital of Sun Yat-Sen University

\section{Yunfeng Pan ( $\nabla$ panyunf@mail.sysu.edu.cn )}

Third Affiliated Hospital of Sun Yat-Sen University https://orcid.org/0000-0001-7459-4970

\section{Research Article}

Keywords: Rheumatoid arthritis, MiRNA, CircRNA, WGCNA, Biological characteristics

Posted Date: February 18th, 2022

DOI: https://doi.org/10.21203/rs.3.rs-1347763/v1

License: (c) (i) This work is licensed under a Creative Commons Attribution 4.0 International License. Read Full License 


\section{Abstract}

BACKGROUND Altered phenotype of Fibroblast-like synoviocyte(FLS) is an important cause of the pathogenesis and progression of rheumatoid arthritis(RA) , but the specific mechanism causing this change has not yet been fully explained. The exact mechanism by which the biological properties of FLS change in RA is still unclear. MiRNAs regulate the biological activity of FLS. Thus, we first used miRNA microarray and WGCNA to confirm the RA-FLS miRNA landscape and establish their biological functions via network analyses at the system level, as well as to provide a platform for modulating the overall phenotypic effects of RA-FLS.

METHODS We enrolled a total of 3 patients with RA and 3 healthy participants, constructed a network analysis of via miRNA microarray and RNA-sequencing. Furthermore, the coexpression analyses of miR-7 and ciRS-7 were verified by siRNA transfection, overexpression and qPCR analyses. Finally, we evaluated the effects of adjusting the expression levels of miR-7 and ciRS-7 on RA-FLS, respectively.

RESULTS We identified distinct miRNA features in RA-FLS, including miR-7, which was significantly lower expressed. Furthermore, we discovered the negative regulatory relationship between ciRS-7 and miR-7 in RA-FLS. Finally, we overexpressed miR-7 in RA-FLS and discovered that miR-7 inhibited RA-FLS hyperproliferation, migration, invasion, and apoptosis, whereas ciRS-7 overexpression reversed these effects.

CONCLUSION Our investigation identified that ciRS-7-miR-7 axis affects the tumor-like biological characters of RA-FLS. These findings could aid in our understanding of essential roles of miR-7 in RA-FLS and will facilitate to development potential intervention target for RA.

\section{Introduction}

Rheumatoid arthritis(RA) is an autoimmune disease with synovitis and joint bone destruction(1). Fibroblast-like synovial cells exist in the synovial tissue of joints, and changes in their proliferation, invasion and other characteristics are important reasons for the pathogenesis and progression of RA(2). In the lesion microenvironment FLSs contributes to joint inflammation and damage by secreting inflammatory mediators, matrix metalloproteinases (MMPs), and invading adjacent cartilage and bone(3). Previous studies have reported that RA-FLS obtained the tumor-like biological characteristics during disease progression(4), presented as hyperproliferation, migration, and invasion(5). Previously reported miRNAs in RA-FLS were predicted from mRNAs based on various bioinformational platform, and these mRNAs were not analyzed at a Weighted Correlation Network Analysis (WGCNA). There are numerous miRNAs can be further investigated in RA-FLS's biological characteristics(6-8). To further identify the miRNAs in RA-FLS, we performed miRNA and mRNA-sequencing on FLS from 3 RA patients, FLS from 3 trauma patients as the control. We identified the key mRNAs through WGCNA platform based on the RNA differential analysis and predicted their target miRNAs. Finally, we intersected the predicted 
miRNAs with the differential miRNAs screened in the miRNA sequencing results, and screened out miR-7 as the key miRNA in RA-FLS.

Circular RNAs (circRNAs) are a special class of non-coding RNA molecules with closed loop structures. Since it is not easily broken down by RNase, its expression is relatively stable(9). However, there are few studies on the roles of circRNAs in the RA-FLS. Cerebellar degeneration-related protein 1 antisense RNA (CDR1as) has more than 70 binding sites for miR-7(10), so it is also known as circular RNA sponge for miR-7 (ciRS-7)(11). Previous studies have shown that ciRS-7 can weaken the downstream regulation of miR-7 after binding with miR-7, thereby indirectly regulating the expression level of downstream genes. (12). Tang et al. (2019) found that compared with healthy people, the expression of ciRS-7 in peripheral blood mononuclear cells (PBMCs) of RA patients was significantly increased, and the expression of miR7 decreased after increasing its expression level (13). In addition, there is increasing evidence that ciRS-7 regulated cell invasion, migration, and proliferation in many cancer types(14-16). Overall, we confirmed a candidate miR-7 and identified that miR-7 inhibits RA-FLS proliferation, migration, and invasion.

Furthermore, we hypothesize that a functional circRNA, ciRS-7, influences the biological properties of RAFLSs, and that this could be due to a microRNA sponging mechanism.

\section{Methods}

\subsection{Patients and tissue samples}

All synovial tissues involved in this study were obtained from patients undergoing joint replacement surgery at the Third Affiliated Hospital of Sun Yat-Sen University, from January 2018 to July 2021. The synovial tissue of the RA group was obtained from 6 patients with active RA, and the diagnostic criteria of the patients were based on the 2010 American College of Rheumatology/European Union Classification of Rheumatology (ACR/EACUR). (4 females and 2 males, 51-67 years of age). The control groups were taken from 4 Patients with knee trauma and no history of knee joint disease, chronic disease and tumor ( 1 females and 3 males, 42-60 years of age). All samples were discarded specimens from the Third Affiliated Hospital of Sun Yat-sen University. This study has been approved by the Ethics Committee of the Third Affiliated Hospital of Sun Yat-sen University, and the approval number of the Ethics Committee is [2020]02-090. All subjects have signed written informed consent for this study in accordance with the Declaration of Helsinki Principles.

\subsection{FLSs culture and characterization}

We isolated FLS from primary synovial tissue using tissue block culture. After culturing between 3rd to 5th generation, FLSs were characterized and used in our study. To identify RA-FLS, we used antibodies against CD68 APC and CD90 FITC (Biolegend, San Diego, CA, USA).

2.3 Microarray and Bioinformatic analysis 
We have performed whole-transcriptome sequencing analysis and the microarray results of IncRNA and mRNA have been deposited in the GEO database (http://www.ncbi.nlm.nih.gov/geo/) for a previous study from the same authors. The accession number is GSE128813. The gene differential expression analysis in this study was performed using the $\mathrm{R}$ language (version 3.5.1). For miRNA sequencing results, miRNAs with $p<0.05$ and $\mid \log 2 \mathrm{FCl} \geq 0.8$ will be screened for further analysis. We carried out functional enrichment analysis of the screened miRNAs by FUNRICH software. The P-value cut-off was set at 0.05 .

For the mRNA sequencing result, according to the number of differential mRNAs, we selected the top 10000 genes to construct the mRNA co-expression network using 'WGCNA' method at Bioinfo Intelligent Cloud (http://www.ehbio.com/Cloud_Platform/front/\#/) to find the modules with good co-expression similarity, whose P-value cut-off was set at 0.05 . To guarantee the scale-free distribution of the network, we choose $\beta=12$ (scale-free R2 = 0.85) as the soft threshold. Next, we transformed the thresholded adjacency matrix into a topological overlap matrix (TOM) and performed hierarchical clustering by genes, and assigned a unique color identifier to each module with dissimilar (dissTOM =1-TOM) topological overlap. To assess the association of gene co-expression modules with RA, we calculated module-trait relationships based on the Pearson correlation between each module and specific phenotype data. The significant co-expression modules were screened out by applying the cut-off of $p<0.05$ and we predicted the miRNAs which can target the genes in each module.

\subsection{Immunofluorescence and imaging}

immunofluorescence was performed on synovial tissue. We embedded the collected synovial tissue with paraffin, trimmed it, and cut it into slices with a thickness of 3-4 $\mu \mathrm{m}$. The tissue was floated in warm water at $40^{\circ} \mathrm{C}$ of the slicer, and then baked in a $60^{\circ} \mathrm{C}$ oven. The sections were successively dewaxed in xylene and anhydrous ethanol, soaked in antigen retrieval buffer, heated in a microwave oven to retrieve antigens, and washed with PBS three times for 5 min each time. Fluorescence quencher and blocking solution were added dropwise, then primary antibody was added dropwise, overnight at $4^{\circ} \mathrm{C}$. The next day, the sections were washed three times with PBS and dried, and incubated with secondary antibody dropwise for $50 \mathrm{~min}$ at room temperature. After washing, DAPI staining solution was added dropwise. After washing again, tissue autofluorescence quencher was added dropwise. After washing three times, the slides were mounted. The fluorescence of synovial tissue was observed under an upright fluorescence microscope.

\subsection{Quantitative real-time reverse transcription PCR}

To validate the selected miRNA and its potential circRNA expression, we performed the RT-qPCR. cDNAs were synthesized using the RT reagent kit with gDNA Eraser (Takara Biotechnology, Tokyo, Japan) and Real-time PCR was performed using TB GreenTMPremix Ex TaqTMII (Takara Biotechnology). GAPDH (AAGGTGAAGGTCGGAGTCAAC [forward]; GGGGTCATTGATGGCAACAATA [reverse]) and U6 were used as internal controls. We performed all qPCR reactions for this study using the ASA4800 fast real-time PCR amplification equipment (Applied Biosystems, Baiyuan gene technology, Suzhou). The relative expression level of each gene was normalized to GAPDH or U6 and calculated by $2-\triangle \triangle \mathrm{Ct}$ method. 


\subsection{Transient transfection and infection.}

We used siRNA to knock down ciRS-7 (RiboBio Inc, Guangzhou, China). The target sequence is GTCTGCAATATCCAGGGTT. Lipo3000 (Invitrogen, California, USA) was used to transfected RA-FLS with ciRS-7 targeting siRNA and the negative control siRNA. Experiments were carried out $48 \mathrm{~h}$ after transfection. For overexpression of miR-7 and ciRS-7, we used lentiviral transductions which were purchased from Shanghai Jikai Gene Co., Ltd. Cell infection was performed according to the lentiviral protocol. We assessed the infection efficiency by analyzing GFP positive rate through flow cytometry. The overexpression efficiency was measured by qPCR.

\subsection{Cell Counting Kit-8}

The proliferation function of RA-FLS was detected by Cell Counting Kit-8 assay (Dojindo, Japan). We took 3-5 generations of overexpressed miR-7, overexpressed ciRS-7 and negative control RA-FLS digested and planted in 96 -well plates, with a density of 20,000 cells/ml plus $100 \mu$ l. Add drop by drop when planting to ensure even distribution. Five duplicate wells were set in each group, and one control well was set with DMEM only. After the cells adhered, $10 \mu \mathrm{l}$ of CCK8 reagent was added at $24 \mathrm{~h}, 48 \mathrm{~h}$, and $72 \mathrm{~h}$, respectively, and the absorbance at $450 \mathrm{~nm}$ was measured with a microplate reader after incubation in the dark for 1.5 h. Draw a line graph of the average absorbance values of different groups at different time points, and compare the absorbance changes of different groups.

\subsection{Cell cycle analysis}

We performed flow cytometry to detect the cell cycle, using the Cell Cycle Detection Kit (KeyGEN BioTECH, Nanjing, China) assay. We took about 50,000 3-5 generations overexpressing miR-7, overexpressing ciRS-7 and negative RA-FLS. After washing once with PBS, the fixed cells were resuspended in $70 \%$ cold ethanol and kept overnight at $4^{\circ} \mathrm{C}$. Next day, we prepared a working solution of RNase A:PI of 1:9 according to the reagent instructions then washed the fixative with PBS. After incubation at room temperature for 60 min in the dark, the cells were washed once again with PBS. Finally, we used flow cytometry to detect the processed RA-FLS. The detection results were analyzed using Modifit software. Proliferation Index (proliferation index, $\mathrm{PI}$ ) represents the cell proliferation activity, $\mathrm{PI}=(\mathrm{S}+\mathrm{G} 2 / \mathrm{M}$ ) phase cell number.

\subsection{Quantification of apoptosis}

To quantify apoptosis, we collected the transferred RA-FLS, washed them with PBS, and treated them with Annexin V-PE/7AAD (Keygen, Nanjing, China) according to the kit instructions. The treated RA-FLS were detected by flow cytometer.

\subsection{Cell migration and invasion assays}

To compare the differences in RA-FLS migration and invasion functions after adjusting miR-7 or ciRS-7, we performed transwell chamber experiments. The small chamber used in the experiment has a pore size 
of $8.0 \mathrm{~mm}$ (Transwell, Corning Labware Products, USA). We washed and resuspended RA-FLS in serumfree medium and seeded them at the upper chamber at a density of $8 \times 104$. The lower chamber was immersed in $600 \mu \mathrm{l}$ of $10 \%$ FBS medium. After completing the above operations, the cells were incubated in a $37^{\circ} \mathrm{C}$ cell incubator. After $48 \mathrm{~h}$, the cells were taken out, the upper chamber was wiped dry, and the cells were fixed with $4 \%$ paraformaldehyde and stained with gentian violet. Finally, observe and count the number of transmembrane cells. For the invasion assay, the specific operation steps are similar to the migration detection. Spread $30 \mu \mathrm{l}$ Matrigel in the upper chamber before seeding the cells in the upper chamber. Matrigel was diluted 1:10 with DMEM and incubated at $37^{\circ} \mathrm{C}$ for $1 \mathrm{~h}$.

\subsection{Statistical analysis}

All statistical data processing in this experiment was performed using SPSS software v25.0. Comparisons between the two groups of data were performed using the $t$ test, and non-normal distributions were performed using the Kruskal-Wallis test. Quantitative data were expressed in the form of mean \pm standard deviation (Mean $\pm S D$ ). The data analysis results of $P<0.05$ were considered to be statistically significant.

\section{Results}

\subsection{Expression profile of mRNAs and miRNAs in RA-FLS and GO analysis}

After sequencing 3 groups of RA patients and 3 groups of healthy patients, we obtained their mRNA and miRNA microarray data. First, we found the mRNA and miRNA with statistical differences in this set of data through differential analysis, which included 3216 differential mRNAs and 97 differential miRNAs. For mRNA screening, we use the $\log F C>1.5$ and $p$-value $<0.05$, and for miRNA screening, we use the $\log F C>0.8$ and $p$-value $<0.05$. We use volcano maps to show different miRNAs and mRNAs (Fig. 1A-B). At the same time, we performed $\mathrm{GO}$ enrichment analysis on mRNAs targeted by differentially expressed miRNAs, The result suggested that these miRNAs are mainly related to Cytoplasm (Cellular component) (Fig. 1C), regulation of nucleobase, nucleoside, nucleotide and nucleic acid metabolism (Biological process) (Fig. 1D), and transcription factor activity (Molecular function) (Fig. 1E).

\subsection{Weighted Co-Expression Network Construction and Key miRNA Identification}

According to the number of differential mRNAs, a total of 10000 genes were selected to conduct the WGCNA. The power of $\beta=12$ (scale-free $R 2=0.85$ ) was selected as the soft-thresholding parameter to conduct a scale-free network (Fig. 2A). 14 modules were identified through hierarchical clustering, representing a collection of genes with different tissue-specific expression patterns (Fig. 2B). We compared the correlation between the various modules (Fig. 2C) as well as the correlation between the modules and RA (Fig. 2D). The blue module and the turquoise module are considered meaningful after identification. We extract the genes of these two modules and predict the miRNAs that target them, and intersect the miRNAs previously screened. In the end, we got the key miRNAs are miR-7 and miR-142-3p (Fig. 2E). Considering that the effect of miR-142-3p on the biological characteristics of RA-FLS had been 
studied(17), we chose miR-7 as the object of further research. Furthermore, we examined miR-7 expression level by RT-qPCR and the results showed its decreased expression in RA-FLS (Fig. 2F).

\subsection{Culturing and identification of FLSs}

Primary fibroblast-like synovial cells crawled out from the edge of synovial tissue through our culturing (Fig. 3A). The third generation of RA-FLS has a unique morphology, which is spindle-shaped and whirlpool-shaped during growth (Fig. 3B). CD90 or CD68 is a marker that distinguishes fibroblasts from synovial cells and macrophages(18). We have detected the positive rate of cultured RA-FLS CD90 as high as $96.6 \%$ by flow cytometry compared with the isotype control group, while the CD 68 test result was negative (Fig. 3C). Numerous studies have demonstrated the correlations between the expression of ciRS7 and miR-7(19-21), Therefore, we decided to find out whether there was a regulation relationship between them in RA-FLS. Immunofluorescence images show that both are expressed in the cytoplasm of RA-FLS and their positions basically overlapped (Fig. 3D).

\section{$3.4 \mathrm{miR}-7 / \mathrm{ciRS}-7$ regulate each other in RA-FLS.}

In order to more intuitively explore the mutual regulation relationship, we use RT-qPCR to detect the relationship between miR-7/ciRS-7 after regulating the expression of one of them. First, we find the ciRS-7 was upregulated in RA-FLS compared with HC-FLSs (Fig. 4A). We then knocked down the ciRS-7 in RAFLS by using siRNA and find that the miR-7 was upregulated in the knockdown group (Fig. 4B). Next, we overexpressed the miR-7/ciRS-7 respectively in RA-FLS by using lentiviral transfection and verified the overexpression efficiency of each group by flow cytometry (Fig. 4C). We found that the expression level of ciRS-7 was downregulated when miR-7 was overexpressed in RA-FLS compared with the negative control group (Fig. 4D-E).

3.5 miR-7/ciRS-7 regulate RA-FLS in proliferation, migration, and invasion

We next explored whether the overexpression of miR-7 and ciRS-7 would affect RA-FLS in cell functions. First, we find that miR-7 overexpression resulted in a significant enhancement in cell proliferation by CCK8 assay (Fig. 5A). Next, flow cytometric analysis detected a significant increase in cell apoptosis and sub-G1 phase in miR-7 overexpression group (Fig. 5B-C). What's more, we found that miR-7 overexpression inhibited RA-FLS in migration and invasion through transwell assay (Fig. 5D-E). We also performed the above experiments on RA-FLS overexpressing ciRS-7, and observed the opposite results.

\section{Discussion}

The main cell group involved in RA synovial tissues is RA-FLS, which exhibits distinct biological characteristics such as hyperproliferation, resistance to apoptosis, and enhanced invasion (22). In recent years, many studies have focused on miRNAs in the study of disease pathogenesis. miRNAs have been shown to be involved in the regulation of a wide variety of biological processes, such as transcription, splicing, and translation (23-25). At the same time, miRNAs have been found to participate in the 
regulation of the pathogenesis and progression of various diseases, including RA(26-28). Previous studies suggested that miR-7 affect the mRNA expression of TNF-a, IL-6, MCP-1 and CCL5 through p65(29). In fact, miR-7 does often play a protective role in various diseases such as inhibiting the growth, invasion, and migration of ovarian cancer by targeting EGFR(30), and inhibiting the progression of osteosarcoma by targeting TAF9B(31). In this study, we first found the core mRNA through WGCNA construction of the differential mRNA in the sequencing results of the RA patient groups and the healthy groups, and then obtained the intersection of the miRNA predicted by the core mRNA and the differential miRNA analyzed by the sequencing results to screen out the RA. The key miRNA was miR-7 and the screening results were verified by qPCR. We did not simply end the study by verifying the expression of miR-7 screened by bioinformatics, but further tried to study its impact on biological functions, so as to more fully explain the role of miR-7 in the pathogenesis and progression of RA impact.

To explore the mechanism of miRNA involved in the regulation of RA, we also studied its classical partner ciRS-7, a newly identified functional circRNA which has been proven to affect the proliferation, migration and invasion of cancer cells in diseases such as gastric cancer, osteosarcoma, melanoma, and hepatocellular carcinoma(21, 32-35). After qPCR, it was found that the expression level of ciRS-7 in RAFLS was higher than that in HC-FLS, and the expression level of miR-7 changed in the opposite direction after regulating ciRS-7, which indicated that ciRS-7 in RA-FLS had an effect on miR-7 Negative regulation of expression. Interestingly, the expression of ciRS-7 also decreased after overexpression of miR-7, so in RA-FLS, miR-7 and ciRS-7 are likely to be negatively regulated by each other. According to recent studies, one of the most important ways in which circRNAs function is as a sponge binding site for miRNAs(36). Therefore, we hypothesized that the combination of ciRS-7 and miR-7 acting as a sponge in RA-FLS negatively regulates each other and modulates the biological properties of RA-FLS. Our cellular function experiments after overexpressing ciRS-7 and miR-7, respectively, showed opposite results, which further verified our conjecture.

In recent years, there have been a lot of studies on the regulation RA-FLS by miRNAs, and these studies have developed from simply exploring the key miRNAs that affect the biological characteristics of RA-FLS to exploring the ways in which these miRNAs affect RA-FLS. For example, The ways in which these miRNAs affect the biological characteristics of RA-FLS include being mediated by exosomes(37), affecting RA-FLS pyroptosis(38), etc. Simply mining a core gene through bioinformatics analysis of sample sequencing results and then conducting cell experiments is certainly convincing. However, since miRNAs are involved in the regulation of diseases in many ways and have a large number of potential binding targets, we should explore its core pathways and downstream targets through further work. Converting the results of cell experiments to help in clinical work also requires a large number of animal experiments and clinical research.

Collectively, our experiments screened miR-7, a key miRNA that affects the biological properties of RAFLS, by bioinformatics technology, and demonstrated the negative regulatory relationship between ciRS-7 and ciRS-7 in RA-FLS. Further, we verified the effect of miR-7/ciRS-7 combination on the proliferation, apoptosis, invasion and other characteristics of RA-FLS through functional experiments. This discovery is 
expected to provide potential targets for the diagnosis and treatment of RA, and has certain value in the exploration of the pathogenesis of RA.

\section{Declarations}

\section{FUNDING}

This work was supported by grants provided from the National Natural Science Foundation of China (No. 81771750).

\section{COMPETING INTERESTS}

The authors declare no conflicts of interest.

\section{AUTHOR CONTRIBUTIONS}

All authors contributed to the study conception and design. Material preparation, data collection and analysis were performed by Yunfeng Pan, Zuoyu Hu and Manli Wang. The first draft of the manuscript was written by Jingrong Chen, Weizhen Weng and Ye Chen. All authors commented on previous versions of the manuscript. All authors read and approved the final manuscript.

\section{ETHICS APPROVAL}

The research was approved by the ethics committee of the Third Affiliated Hospital at the Sun Yat-sen University and all subjects were given the written informed consent in accordance with the Declaration of Helsinki. The Ethics board approval number is [2020]02-090.

\section{References}

1. Scott DL, Wolfe F and Huizinga TWJ (2010) Rheumatoid arthritis. Lancet (London, England) 376, 10941108

2. Nygaard G and Firestein GS (2020) Restoring synovial homeostasis in rheumatoid arthritis by targeting fibroblast-like synoviocytes. Nature reviews. Rheumatology 16, 316-333

3. Rodriguez-Trillo A, Mosquera N, Pena C et al (2020) Non-Canonical WNT5A Signaling Through RYK Contributes to Aggressive Phenotype of the Rheumatoid Fibroblast-Like Synoviocytes. Frontiers in immunology 11,555245

4. Guiducci S, Del Rosso A, Cinelli M et al (2005) Rheumatoid synovial fibroblasts constitutively express the fibrinolytic pattern of invasive tumor-like cells. Clin Exp Rheumatol 23, 364-372

5. Liu Y, Pan YF, Xue YQ et al (2018) UPAR promotes tumor-like biologic behaviors of fibroblast-like synoviocytes through PI3K/Akt signaling pathway in patients with rheumatoid arthritis. Cell Mol Immunol 
6. Ren C, Li M, Zheng Y, Wu F, Du W and Quan R (2021) Identification of diagnostic genes and vital microRNAs involved in rheumatoid arthritis: based on data mining and experimental verification. PeerJ 9 , e11427

7. Nakamachi Y, Kawano S, Takenokuchi M et al (2009) MicroRNA-124a is a key regulator of proliferation and monocyte chemoattractant protein 1 secretion in fibroblast-like synoviocytes from patients with rheumatoid arthritis. Arthritis Rheum 60, 1294-1304

8. Renman E, Brink M, Ärlestig L, Rantapää-Dahlqvist S and Lejon K (2021) Dysregulated microRNA expression in rheumatoid arthritis families-a comparison between rheumatoid arthritis patients, their firstdegree relatives, and healthy controls. Clin Rheumatol 40, 2387-2394

9. Enuka Y, Lauriola M, Feldman ME, Sas-Chen A, Ulitsky I and Yarden Y (2016) Circular RNAs are longlived and display only minimal early alterations in response to a growth factor. Nucleic Acids Res 44, $1370-1383$

10. Guo Z, Cao Q, Zhao Z and Song C (2020) Biogenesis, Features, Functions, and Disease Relationships of a Specific Circular RNA: CDR1as. Aging and disease 11, 1009-1020

11. Memczak S, Jens M, Elefsinioti A et al (2013) Circular RNAs are a large class of animal RNAs with regulatory potency. Nature 495, 333-338

12. Yang $X$, Ye T, Liu H et al (2021) Expression profiles, biological functions and clinical significance of circRNAs in bladder cancer. Molecular cancer 20, 4

13. Tang X, Wang J, Xia X et al (2019) Elevated expression of ciRS-7 in peripheral blood mononuclear cells from rheumatoid arthritis patients. Diagnostic pathology 14,11

14. Jiang C, Zeng X, Shan R et al (2020) The Emerging Picture of the Roles of CircRNA-CDR1as in Cancer. Frontiers in cell and developmental biology 8, 590478

15. Zhang F, Xu Y, Ye W, Jiang J and Wu C (2020) Circular RNA S-7 promotes ovarian cancer EMT via sponging miR-641 to up-regulate ZEB1 and MDM2. Biosci Rep 40

16. Liu L, Liu FB, Huang M et al (2019) Circular RNA ciRS-7 promotes the proliferation and metastasis of pancreatic cancer by regulating miR-7-mediated EGFR/STAT3 signaling pathway. Hepatobiliary Pancreat Dis Int 18, 580-586

17. Qiang J, Lv T, Wu Z and Yang X (2019) Down-regulation of microRNA-142-3p inhibits the aggressive phenotypes of rheumatoid arthritis fibroblast-like synoviocytes through inhibiting nuclear factor-kappaB signaling. Biosci Rep 39 
18. Bi X, Guo XH, Mo BY et al (2019) LncRNA PICSAR promotes cell proliferation, migration and invasion of fibroblast-like synoviocytes by sponging miRNA-4701-5p in rheumatoid arthritis. EBioMedicine

19. Han JY, Guo S, Wei N et al (2020) ciRS-7 Promotes the Proliferation and Migration of Papillary Thyroid Cancer by Negatively Regulating the miR-7/Epidermal Growth Factor Receptor Axis. Biomed Res Int 2020, 9875636

20. Dou Z, Gao L, Ren W et al (2020) CiRS-7 functions as a ceRNA of RAF-1/PIK3CD to promote metastatic progression of oral squamous cell carcinoma via MAPK/AKT signaling pathways. Exp Cell Res 396, 112290

21. Zhou X, Li J, Zhou Y et al (2020) Down-regulated ciRS-7/up-regulated miR-7 axis aggravated cartilage degradation and autophagy defection by PI3K/AKT/mTOR activation mediated by IL-17A in osteoarthritis. Aging (Albany NY) 12, 20163-20183

22. Wang M, Chen Y, Bi X et al (2021) LncRNA NEAT1_1 suppresses tumor-like biologic behaviors of fibroblast-like synoviocytes by targeting the miR-221-3p/uPAR axis in rheumatoid arthritis. J Leukoc Biol

23. Cho WC (2007) OncomiRs: the discovery and progress of microRNAs in cancers. Mol Cancer 6, 60

24. Tsuchiya S, Okuno Y and Tsujimoto G (2006) MicroRNA: biogenetic and functional mechanisms and involvements in cell differentiation and cancer. J Pharmacol Sci 101, 267-270

25. Scaria V, Hariharan M, Maiti S, Pillai B and Brahmachari SK (2006) Host-virus interaction: a new role for microRNAs. Retrovirology 3, 68

26. Zhou SS, Jin JP, Wang JQ et al (2018) miRNAS in cardiovascular diseases: potential biomarkers, therapeutic targets and challenges. Acta Pharmacol Sin 39, 1073-1084

27. Marques-Rocha JL, Samblas M, Milagro FI, Bressan J, Martínez JA and Marti A (2015) Noncoding RNAs, cytokines, and inflammation-related diseases. Faseb j 29, 3595-3611

28. Sugatani T, Vacher J and Hruska KA (2011) A microRNA expression signature of osteoclastogenesis. Blood 117, 3648-3657

29. Wen X, Chen X, Liang X et al (2018) The small molecule NSM00191 specifically represses the TNFa/NF-KB axis in foot and ankle rheumatoid arthritis. Int J Biol Sci 14, 1732-1744

30. Cui X, Song K, Lu X, Feng W and Di W (2021) Liposomal Delivery of MicroRNA-7 Targeting EGFR to Inhibit the Growth, Invasion, and Migration of Ovarian Cancer. ACS Omega 6, 11669-11678

31. Gu W, Chen P, Ren P, Wang Y, Li X and Gong M (2021) Downregulation of TAF9B by miR-7-5p Inhibits the Progression of Osteosarcoma. Onco Targets Ther 14, 2917-2927 
32. Pan H, Li T, Jiang Y et al (2018) Overexpression of Circular RNA ciRS-7 Abrogates the Tumor Suppressive Effect of miR-7 on Gastric Cancer via PTEN/PI3K/AKT Signaling Pathway. J Cell Biochem $119,440-446$

33. Xu B, Yang T, Wang Z, Zhang Y, Liu S and Shen M (2018) CircRNA CDR1as/miR-7 signals promote tumor growth of osteosarcoma with a potential therapeutic and diagnostic value. Cancer Manag Res 10, 4871-4880

34. Zhang L, Li Y, Liu W, Li H and Zhu Z (2018) Analysis of the complex interaction of CDR1as-miRNAprotein and detection of its novel role in melanoma. Oncol Lett 16, 1219-1225

35. Chen L, Shi J, Wu Y et al (2020) CircRNA CDR1as promotes hepatoblastoma proliferation and stemness by acting as a miR-7-5p sponge to upregulate KLF4 expression. Aging (Albany NY) 12, 1923319253

36. Kristensen LS, Andersen MS, Stagsted LVW, Ebbesen KK, Hansen TB and Kjems J (2019) The biogenesis, biology and characterization of circular RNAs. Nat Rev Genet 20, 675-691

37. Zhang J, Zhang Y, Ma Y, Luo L, Chu M and Zhang Z (2021) Therapeutic Potential of Exosomal circRNA Derived from Synovial Mesenchymal Cells via Targeting circEDIL3/miR-4853p/PIAS3/STAT3/VEGF Functional Module in Rheumatoid Arthritis. Int J Nanomedicine 16, 7977-7994 38. Chen S, Luo Z and Chen X (2021) Hsa_circ_0044235 regulates the pyroptosis of rheumatoid arthritis via MiR-135b-5p-SIRT1 axis. Cell Cycle 20, 1107-1121

\section{Figures}


A

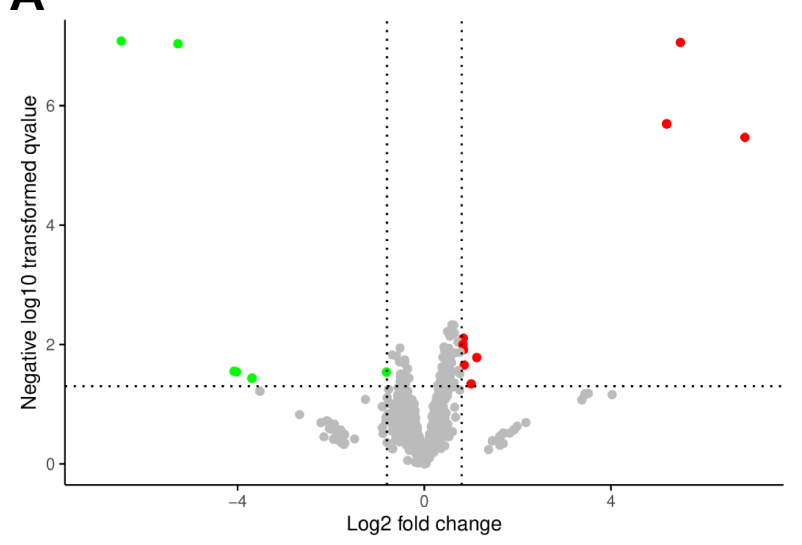

C

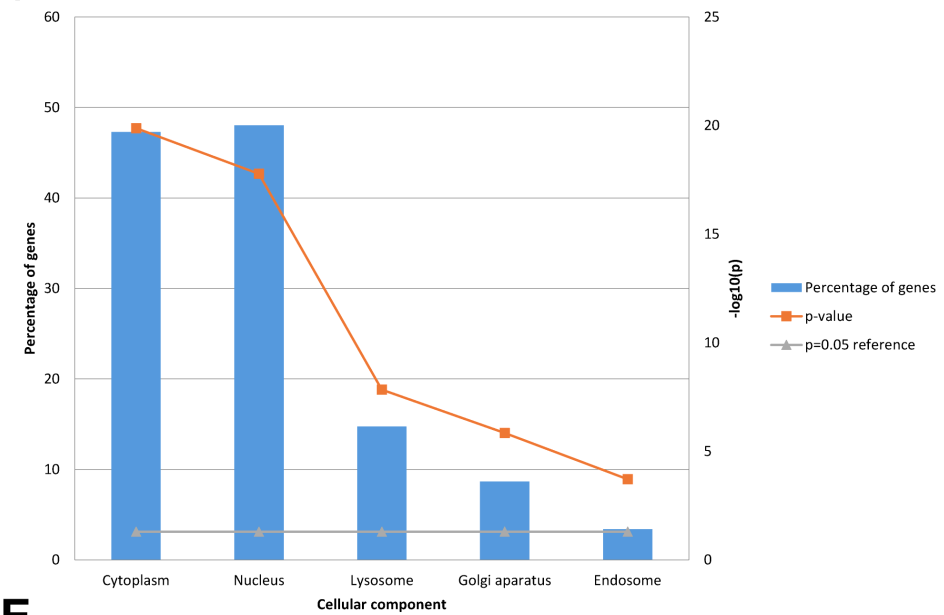

$\mathbf{E}$

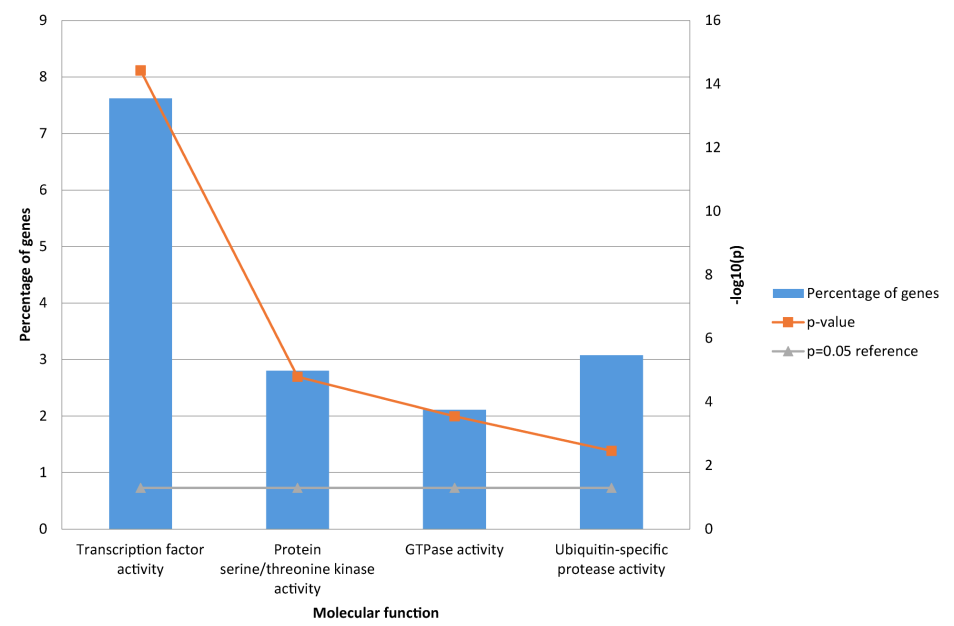

B

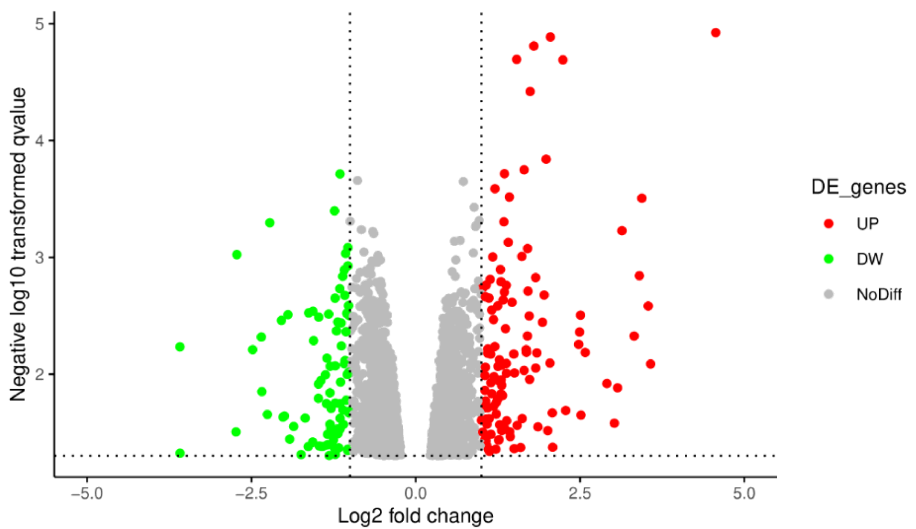

D

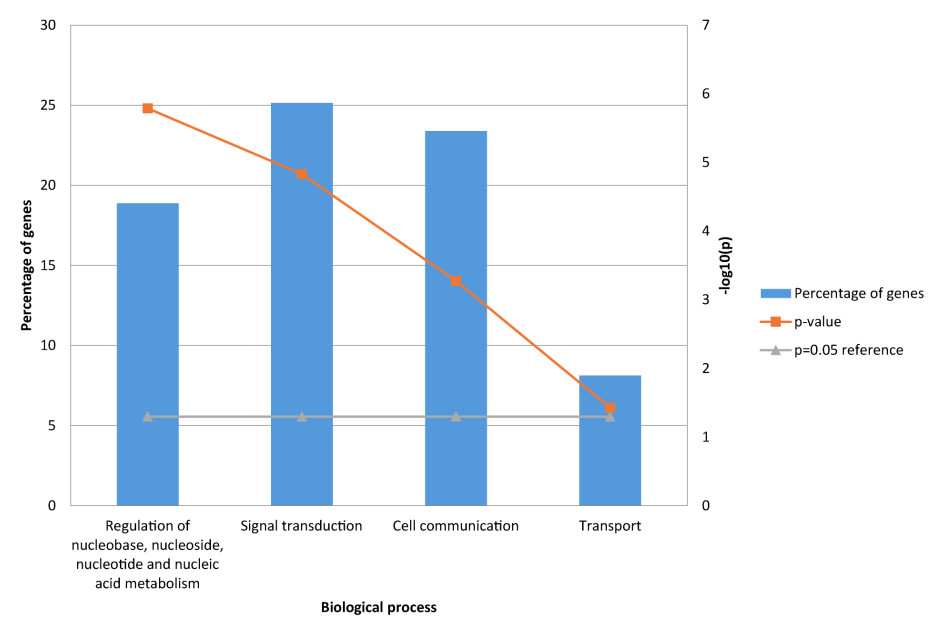

Figure 1

Differential gene volcano map and functional enrichment

A. Volcano plot of differentially expressed miRNAs $(P<0.05, \log F C>0.8) \otimes B$. Volcano plot of differentially expressed mRNAs $(P<0.05)$; C-E. GO analysis of differentially expressed miRNAs $(P<0.05)$ 


\section{Figure 2}

WGCNA analysis of the differentially expressed mRNA
A. Detection of the soft-thresholding powers;
B. WGCNA module plot;
C. WGCNA module correlation plot;
D. WGCNA module trait correlation plot; $E$. Venn diagram for screened module and differentially expressed miRNA; F. Differences in miR-7 expression between normal group and RA group measured by qPCR. All the results were presented as the mean \pm standard deviation (S.D.) based on 3 replicates involving 3 samples. ${ }^{*} \mathrm{P}<0.05,{ }^{*} \mathrm{P}<0.01$, and ${ }^{*} * \mathrm{P}<0.001$ versus control groups.

A

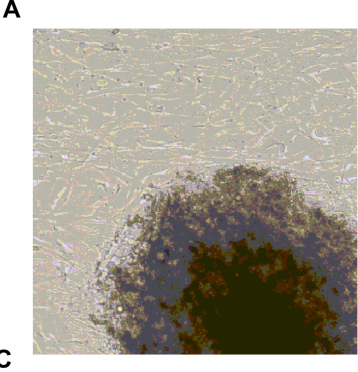

c

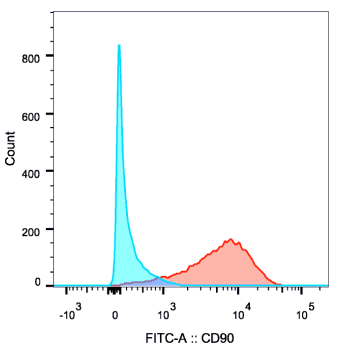

B
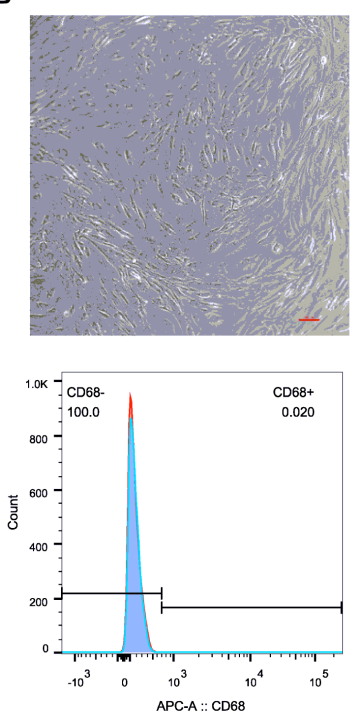

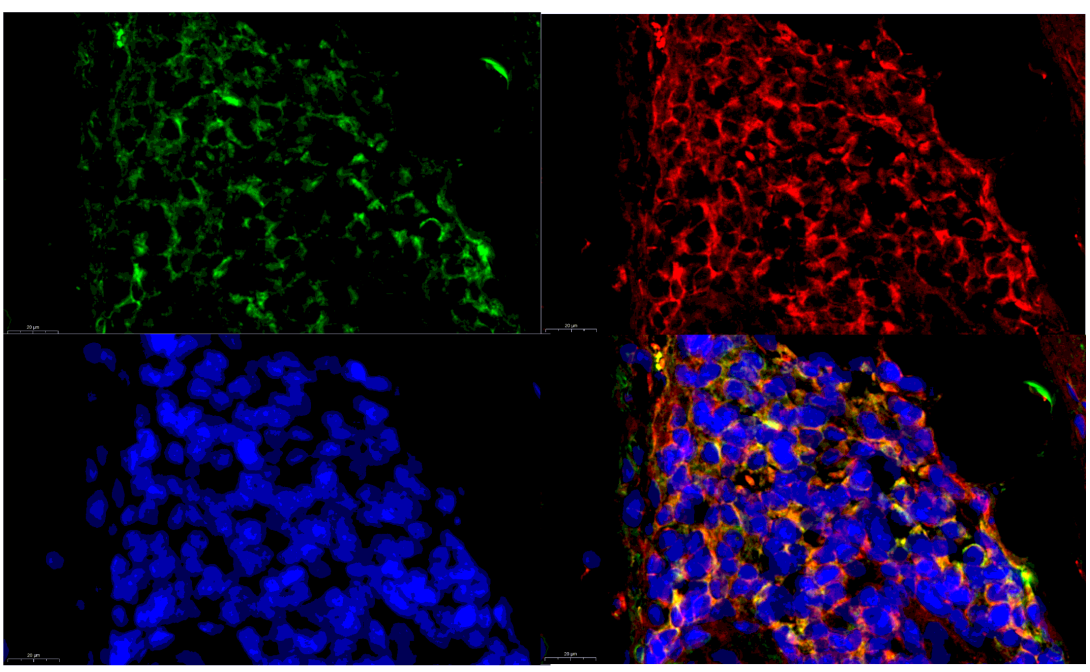

\section{Figure 3}

Primary RA-FLS culture and preliminary identification of miR-7/ciRS-7

A. RA-FLS crawled out of the synovial tissue block; B. The 3rd generation RA-FLS had a unique shape; C. CD90-positive and CD68-negative cells were identified as RA-FLSs; D. CiRS-7 in synovial tissue was recognized as red by FISH probe; MiR-7 in synovial tissue was recognized as green by antibodies; The nucleus was stained blue by DAPl; After the merge, it could be found that the distribution of miR-7 and ciRS-7 overlapped in the synovial tissue

\section{Figure 4}

miR-7 and ciRS-7 negatively regulate each other

A. ciRS-7 was upregulated in RA-FLSs compared with HC-FLSs; B.knockdown efficiency of ciRS-7 and miR-7 was upregulated in ciRS-7 knockdown groups; C. RA-FLSs in each group were transfected with 
Lentiviral and the GFP transfection efficiency was analysed by flow cytometry; D. Reverse transcriptase (RT) - qPCR was used to evaluate the expression efficiency of miR-7 and ciRS-7; E. ciRS-7 was downregulated in miR-7 overexpression groups. ${ }^{*} P<0.05,{ }^{*} \mathrm{P}<0.01$, and ${ }^{*} * \mathrm{P}<0.001$ versus control groups.

\section{Figure 5}

The functional experiments of RA-FLSs in which miR-7 or ciRS-7 was overexpressed

A. The effect of miR-7 or ciRS-7 overexpression on RA-FLS proliferation measured by the CCK8 assay; $\mathbf{B}$. The effect of miR-7 or ciRS-7 overexpression on RA-FLS cycle; C. The effect of miR-7 or ciRS-7 overexpression on RA-FLS apoptosis; D-E. The effect of miR-7 or ciRS-7 overexpression on RA-FLS migration and invasion. All the results were presented as the mean \pm standard deviation (S.D.) based on 3 replicates involving 3 samples. ${ }^{\star} \mathrm{P}<0.05$, ${ }^{\star *} \mathrm{P}<0.01$, and ${ }^{\star \star *} \mathrm{P}<0.001$ versus control groups. 